\title{
Face Detection Using Bionic Cascaded Framework
}

\author{
Jin $\mathrm{Li}^{(\bowtie)}$, Ziyue Chen, Shunxin Ouyang, Jingyu Xie, \\ Yue $\mathrm{Hu}$, and Hui Lv \\ Hubei University of Technology, \\ Wuhan, Hubei, People's Republic of China \\ lijin@hbut.edu.cn
}

\begin{abstract}
Face interaction plays an irreplaceable role in the service robots human-robot interaction, while face detection in this kind of scenario are challenging due to restrictions on computing capabilities and power, the character of real-time and requirements of the interaction pattern. Recent studies show that deep learning approaches can achieve impressive performance on these kinds of tasks. Therefore, a bionic cascaded framework adopted a cascaded structure with two stages of carefully designed face detectors is proposed in this paper, which exploits saccade and attention mechanism of human eyes to balance the performance. In addition, in the working process of the service robots, a new online sampling strategy that can improve the performance of interaction patterns is presented. In this way, the real time face detection and more natural human-robot interaction pattern can be achieved in service robot systems.
\end{abstract}

Keywords: Face detection · Human-robot interaction •

Bionic cascaded framework

\section{Introduction}

Face detection is essential to many applications, such as face recognition, facial expression analysis and human-robot interaction, while the large visual variations of faces, including occlusions, large pose variations and extreme lightings, impose great challenges for its applications. In some special scenario such as the service robots, some special requirements of computing capabilities, power limit, the character of real-time and the natural interaction pattern become new challenges.

Viola Jones Face Detector, a kind of cascade face detector, is first proposed by Viola and Jones [1], which utilizes Haar-Like features and AdaBoost to train cascaded classifiers. They have presented an approach for face detection to minimize the computation time and achieve high detection accuracy. The approach was used to construct a faster face detection system than the previous approach, which could be used to construct highly efficient detectors for other objects as well. Because its low computational complexity, good performance with real-time efficiency can be achieved, which is suitable for embedded system application such as service robots. However, although the Viola Jones Face Detector has excellent real-time capability, the detector may 
degrade significantly in practical applications. In order to solve the problem mentioned above, many subsequent studies pay attentions to more powerful learning algorithms.

For example, Yang et al. [2] proposed that the feature representation used for face detection still cannot meet the demand for effectively and efficiently handling faces with large appearance variance in the wild. They borrow the concept of channel features to the face detection domain, which extends the image channel to diverse types such as gradient magnitude and oriented gradient histograms and therefore encodes rich information in a simple form. They adopt a novel variant called aggregate channel features, make a full exploration of feature design, and discover a multi-scale version of features with better performance. A multi-view detection approach is also proposed with consideration of featuring score re-ranking and detection adjustment to deal with facial poses in the wild. Pham et al. [3] present a method that extends the integral image to do fast integration over the interior of any polygon that is not necessarily rectilinear and apply the method to Viola and Jones' object detection framework. The polygonal Haar-like features are proposed to improve classical Haar-like features. Zhu et al. [4] integrate the cascade-of-rejectors approach with the Histograms of Oriented Gradients (HoG) features to achieve a fast and accurate human detection system.

Even with more advanced features and classifiers, the detection accuracy may also degrade significantly in the complex real environment. Thus, some works are done to improve the performance as well.

Mathias et al. [5] show a properly trained vanilla DPM that improves over commercial and research systems and a detector based on rigid templates-similar in structure to the Viola \& Jones detector, which can reach similar performance. Yan et al. [6] solve the speed bottleneck of deformable part model (DPM), while maintaining the accuracy in detection on challenging datasets. A proximal gradient algorithm is adopted to progressively learn the low rank filter in a discriminative manner. For cascade part pruning, neighborhood aware cascade is proposed to capture the dependence in neighborhood regions for aggressive pruning. Look-up tables are constructed to replace expensive calculations of orientation partition and magnitude with simpler matrix index operations for HOG feature extraction. Zhu et al. [7] present a unified model for face detection, pose estimation, and landmark estimation in real-world, cluttered images. The model is based on a mixture of trees with a shared pool of parts and the results show that tree-structured models are surprisingly effective at capturing global elastic deformation, while being easy to optimize unlike dense graph structures.

Although the DPM for face detection achieve remarkable performance, they need high computational expense, which limits their application in service robots.

Recently, convolutional neural networks (CNNs) develop fast and are widely used in the computer vision tasks such as image classification [8], face detection and face recognition [9]. Recent researches proved that CNNs can achieve high performance in these kinds of tasks. Some CNNs based face detection approaches have been proposed in recent years.

Yang et al. [10] propose a novel deep convolutional network (DCN) that achieves outstanding performance on FDDB, PASCAL Face, and AFW. They consider finding faces from a new perspective through scoring facial parts responses by their spatial structure and arrangement. The scoring mechanism is carefully formulated considering challenging cases where faces are only partially visible. The network can detect faces 
under severe occlusion and unconstrained pose variation, which are the main difficulty and bottleneck of the face detection approaches above. Li et al. [11] propose a cascade architecture built on convolutional neural networks with very powerful discriminative capability, while maintaining high performance. The proposed CNN cascade operates at multiple resolutions, quickly rejects the background regions in the fast-low resolution stages, and carefully evaluates a small number of challenging candidates in the last high-resolution stage. To improve localization effectiveness, and reduce the number of candidates at later stages, a CNN-based calibration stage is introduced after each of the detection stages in the cascade. The proposed method achieves high detection performance on two public face detection benchmarks. CNN often need high computing resource, so some lighter and faster face detectors such as the MTCNN proposed in reference [12] and the LFFD proposed in reference [13] are developed. While general speaking, the computation load of CNN-based method is relatively high for an embedded system such like a service robot, so it's performances of real-time and energy consumption need to be improved.

In this paper, a bionic cascaded framework is proposed which exploits saccade and attention mechanism of human eyes to balance the performance. Our framework adopts a cascaded structure with one stage of vision attention and two stages of carefully designed cascaded detectors which can balance the performance and the costs. In addition, in the working process of the service robots, we propose a new online sampling strategy that can improve the performance of interaction patterns. Our method achieves real time face detection in service robot systems, and more natural humanrobot interaction pattern.

\section{Approach}

In this section, we will describe our approach towards face detection and alignment by a bionic cascaded framework.

\subsection{Overall Framework}

The overall pipeline of our approach is shown in Fig. 1. Given an image as a frame the service robot has got, we initially send it into an attention captor to build an image pyramid in different scales, which is the input of the following two-stage cascaded detector.

Stage 1: Firstly, we introduce a unit named attention captor to deal with the frame. The attention captor focuses on parts of the image randomly, and generates a set of images including different parts of the original image, which forms an image pyramid. The pyramid contains different scales of the original image. The following detector modules will find patterns like face in this dynamic pyramid.

Stage 2: The second module is a Front Detector. Front Detector is a fast and light face detector. It is used to detect whether faces exist in the fragment the attention captor gets from the image in the field of view. Since the pyramid generated by the attention captor contains many sub-images, the Front Detector should have the capability of process pictures fast, and using limited computational power, which is important for an 


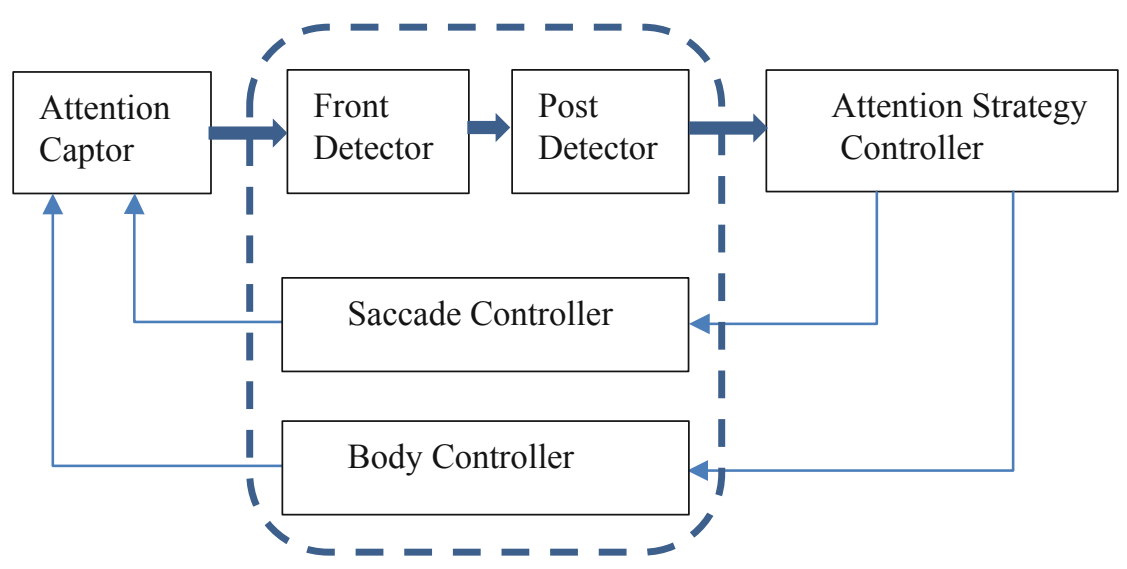

Fig. 1. The overall pipeline of the bionic cascaded framework.

embedded system to realize a real-time process. When the results of the Front Detector are given, the robot should choose one of the faces to establish eye contact at a particular moment.

Stage 3: The third module is a Post Detector. General speaking, a light and fast face detector has a relatively low accuracy. So, the result of the Front Detector should be confirmed by the Post Detector which has higher performance. The output of the Post Detector should be regards as faces. The Post Detector may need more computing resources and longer time, while the point is that it only deals with the sub-images generated by the Front Detector, which are more likely including faces. The number of this kind of sub-images is limited. In fact, the Motion Control Unit including Saccade Controller and Body Controller already moves to focus on one selected face when the Front Detector gives the results, which make the robot seem agile. The Post Detector and the eye contact process at the same time, and when the face is confirmed, the eye contact will continue, if it is proved that the sub-image does not contain a face, the robot may move its attention to another zone of the image.

Stage 4: The results of the cascaded detector is sent to the module of the Attention Strategy Controller. The Attention Strategy Controller control the behavior of the human-robot interaction, according to the information of faces detected by the detector. The Attention Strategy Controller may control the robot focus on one face, switch to another face, or move to a new area to search something new. When the action is decided, the Attention Strategy Controller will send control information to the execution units including the Saccade Controller and Body Controller. The small amplitude motion may be executed by the Saccade Controller and the greater amplitude motion may be executed by the Body Controller.

Stage 5: The decision of the Attention Strategy Controller may be executed by the Saccade Controller and Body Controller. The Saccade Controller controls the rapid and jerky movement of the eyes between positions of rest, which realized by driving the eyeballs of the robot. Saccade is a characteristic pattern of human eye movements. This design makes a more human-like robot when it communicates with people. What's 
more, the saccade is related to the attention shift, so this mechanism may bring a more human-like information processing pattern at the same time. When the moving range of focus exceeds the saccade, the Body Controller will drive the head or body to move. The moving of the focus affects the images captured by Attention Captor in turn.

\subsection{The Implementation of Modules}

Attention Captor: Attention Captor captures segments from the whole image. The size and location of the sub-image is random, which ensures that every part of the image may be observed. There are two style of method to capture sub-image. One is uniform distribution method, which capture every part of the image in the same probability. The other is normal distribution method, namely, the area close to a particular position has the higher probability to be captured, which means that the attention is focusing on a particular part of the image, normally speaking the center of the picture.

The Front Detector: As mentioned above, the Front Detector should be fast and light, and the accuracy is not so important. It should be run on a embedded system efficiently. Considering the above factors, we choose Viola Jones Face Framework to design the Front Detector. The Viola Jones face detection [1] classifies images based on the value of three kinds of rectangle features as shown in Fig. 2.
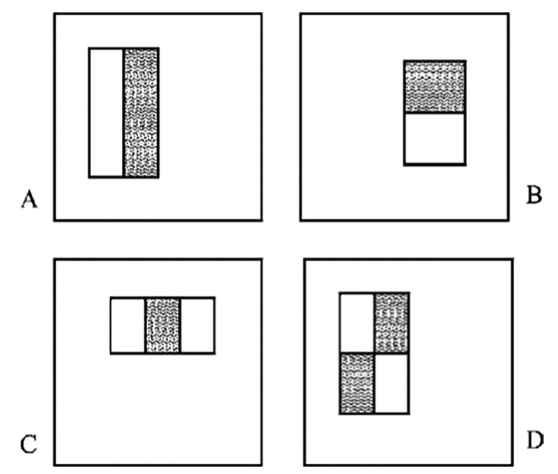

Fig. 2. Three kinds of rectangle features. Figure (A) and (B) show the two-rectangle features. Figure (C) shows a three-rectangle feature, and (D) shows a four-rectangle feature.

The rectangle features above can be computed very rapidly using an intermediate representation for the image which called the integral image [14]. The integral image at location $\mathrm{x}, \mathrm{y}$ contains the sum of the pixels above and to the left of $\mathrm{x}, \mathrm{y}$.

A cascade of classifiers is constructed which achieves increased detection performance while radically reducing computation time. The key insight is that smaller, and therefore more efficient, boosted classifiers can be constructed which reject many of the negative sub-windows while detecting almost all positive instances. Simpler classifiers 
are used to reject the majority of sub-windows before more complex classifiers are called upon to achieve low false positive rates.

The Post Detector: As mentioned above, the Post Detector should have high performance and accuracy. So, in this paper, we choose the Multi-task Cascaded Convolutional Networks to build the post detector. This framework adopts a cascaded structure with three stages of carefully designed deep convolutional networks that predict face and landmark location in a coarse-to-fine manner. In addition, in the learning process, a new online hard sample mining strategy is proposed which can improve the performance automatically without manual sample selection. This method can achieve very fast speed in joint face detection and alignment and has relatively low computational complexity when performing inference.

Attention Strategy Controller: The Attention Strategy Controller determine the position of the focus on random time intervals. It makes the robots act like a human observing behavior. As we know, saccade is a specific behavior in human visual system. When we make a saccade, our visual system is blind during the flight of the saccade, so we can see at the beginning and we can see at the end but while the eye is moving, we cannot see. The Attention Strategy Controller controls the Saccade Controller, Body Controller and the Attention Captor to model this kind of human visual behavior and attention mechanism. The Attention Strategy Controller achieves similar features in average fixation duration, average saccade amplitude and fixation counts. Interest area locates in the faces with higher probability, which matches the visual instinct of human.

\section{Experiments}

In this section, we evaluate the effectiveness of the proposed Bionic Cascaded Framework, and test the system in three aspects: the random pyramids generating, the cascaded face detector and the attention distribution. The system is implemented by Python 3.5 in TensorFlow framework.

\subsection{The Random Pyramids Generating}

When a frame of image is obtained, the Attention Captor captures segments from the whole image. The size and location of the sub-image is random, and generate a set of sub-images including different parts of the original image, then an image pyramid is formed after normalization. A test image is shown in Fig. 3. 


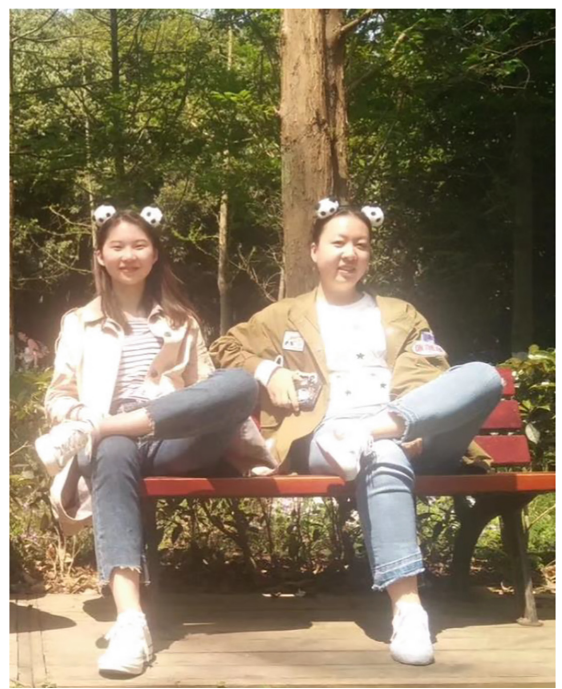

Fig. 3. A frame of image.

The sub-images captured by the Attention Captor and the random pyramid generated are shown in Figs. 4 and 5 respectively.
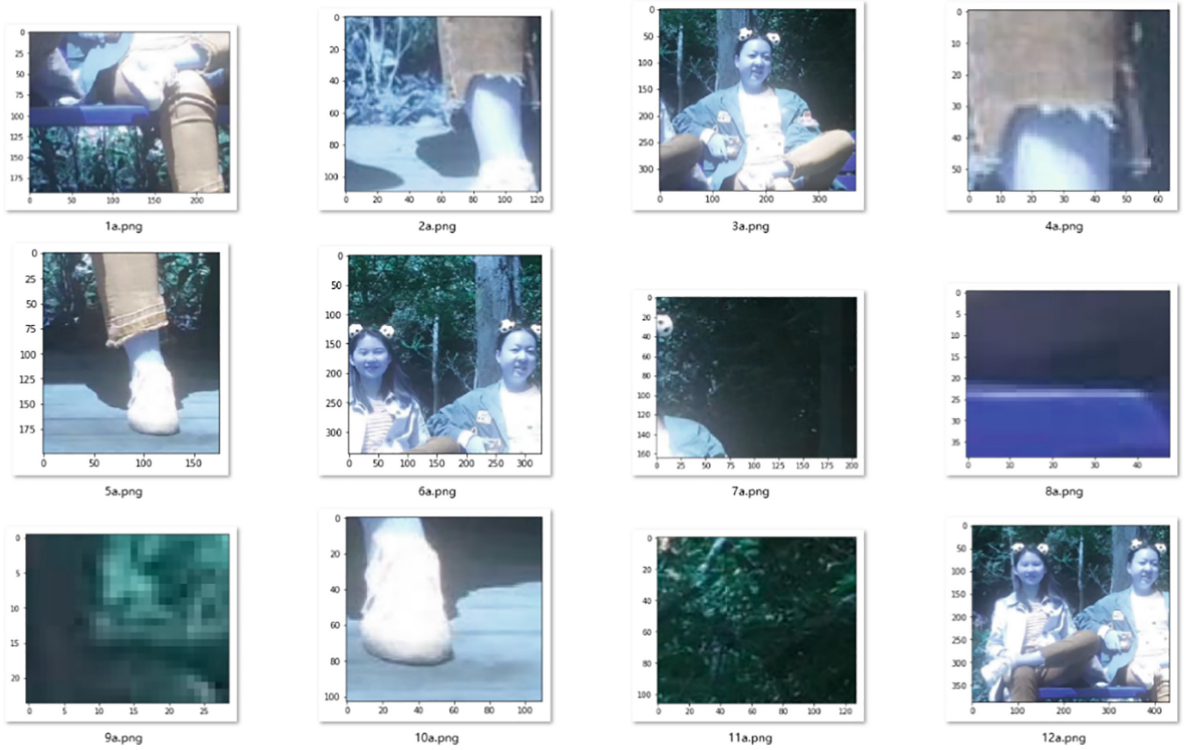

Fig. 4. The sub-images captured by the attention captor. 

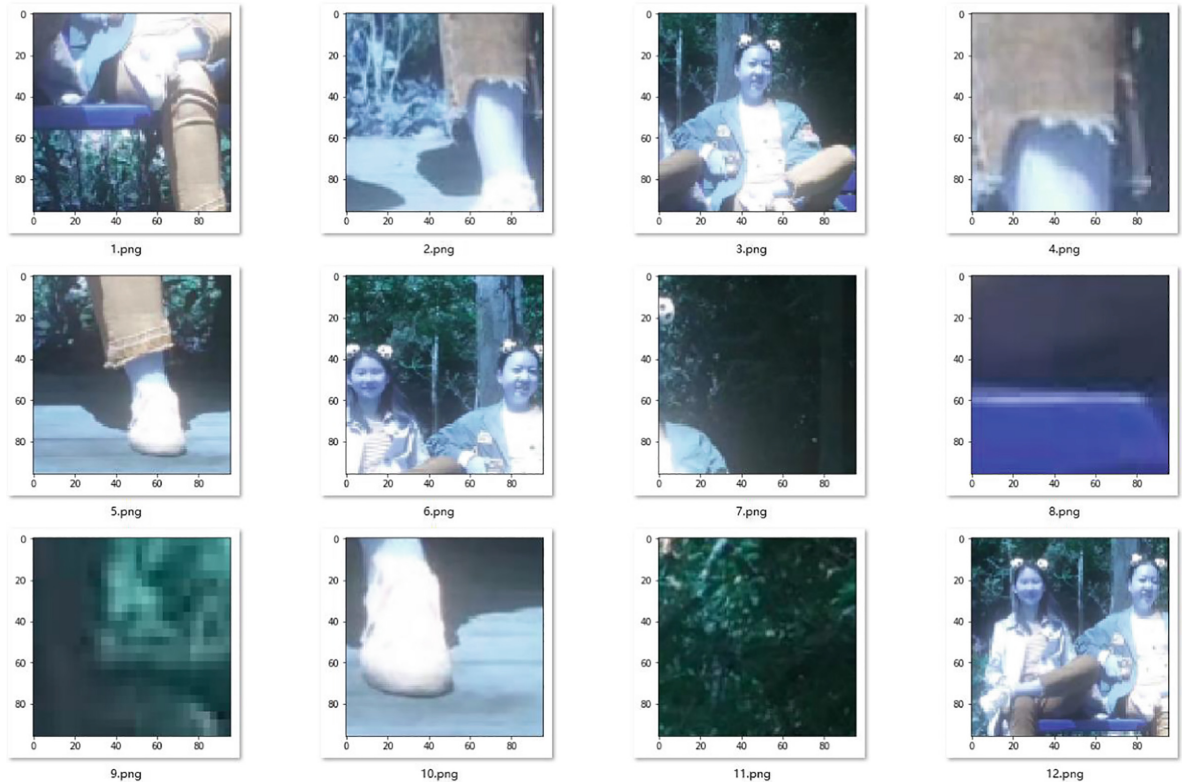

Fig. 5. The random pyramid.

We can see that the sub-image may cover any part of the frame, and the pyramid contains different scales of the original image. The following detector modules will find patterns like face in this dynamic random pyramid.

\subsection{The Cascaded Face Detector}

The dynamic random pyramid generated by the Attention Captor is passed into the Front Detector which is driven by Viola Jones Face Framework. Then the result is passed into the Post Detector which is driven by the Multi-task Cascaded Convolutional Networks to confirm. This procedure is shown in Fig. 6.

We can see that sometimes the faces proposed by the Front Detector can be confirmed by the Post Detector, while the bounding box may be adjusted. Sometimes, the sub-image proposed by the Front Detector contains no face in fact, and the sub-image will be rejected by the Post Detector. It proves that the Post Detector has higher accuracy than the Front Detector, and the cascade detector works. 

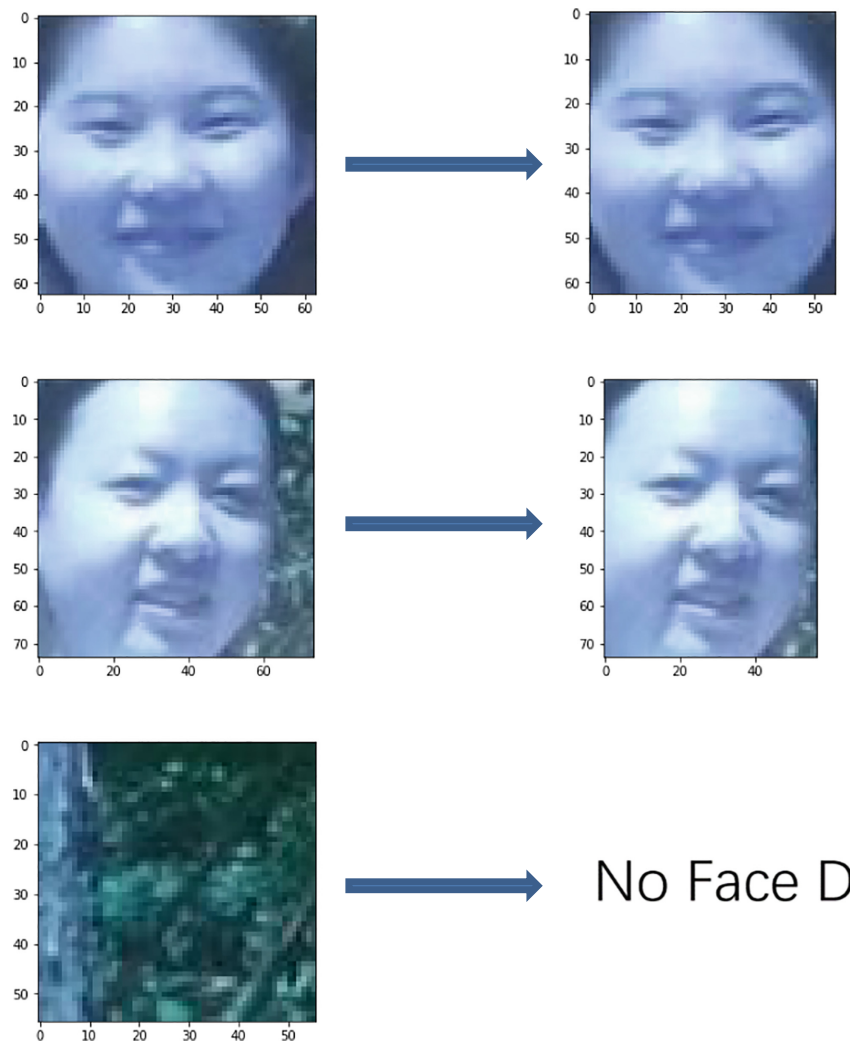

\section{No Face Detected}

(A) Proposed by the Front Detector

(B) Confirmed by the Post Detector

Fig. 6. The cascaded face detector.

\subsection{The Attention Distribution}

Saccade is a specific behavior in human visual system. When we make a saccade, our visual system is blind during the flight of the saccade, so we can see at the beginning and we can see at the end but while the eye is moving, we cannot see. And saccade is considered to have relationship with attention shift. Eye tracking data can show the process of attention drifting when human observing, as shown in Fig. 7 [15]. 


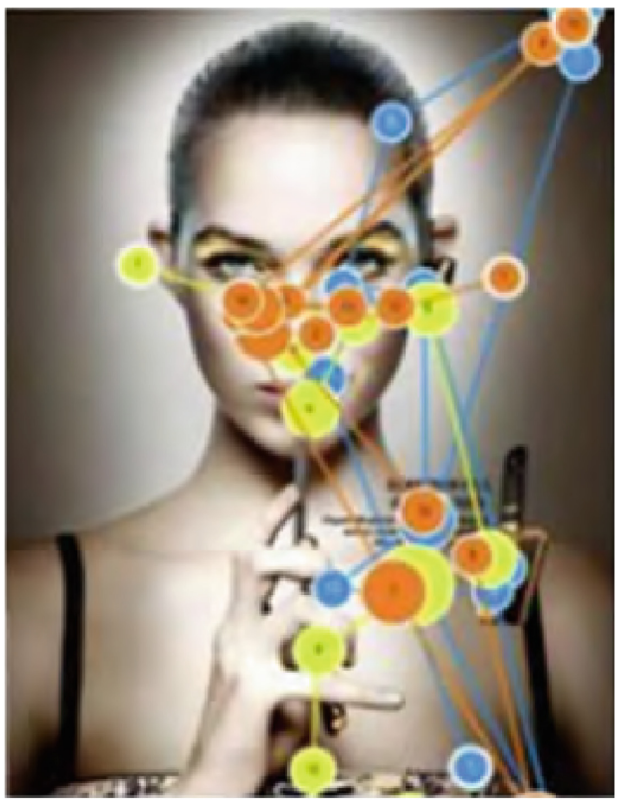

(A)
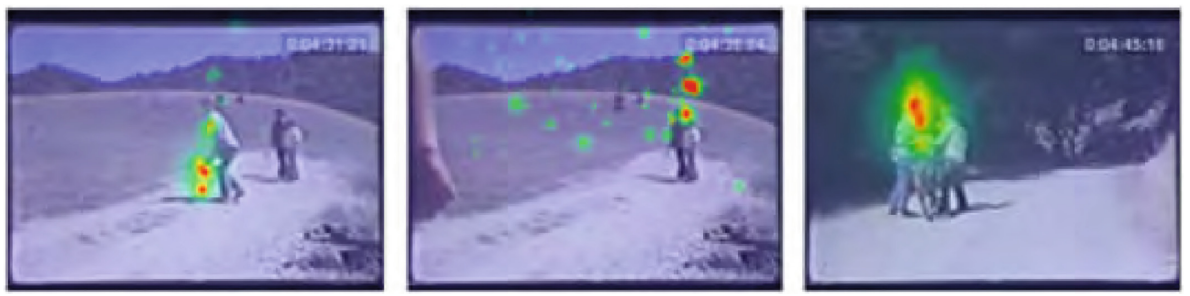

(B)

Fig. 7. Visualization of the eye tracking.

We can see that the areas containing faces are easy to attract the attention of the observers. We got similar results in our experiment using the Attention Strategy Controller, as shown in Fig. 8.

It's shown that the robot tends to focus on the faces just like humans do. This will help the service robots to act and observe in the similar way as human beings, which could improve the performance of interaction patterns. 


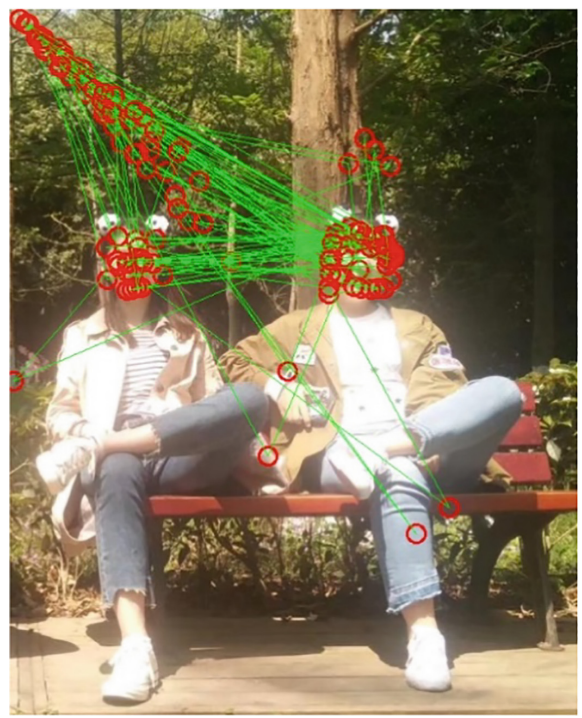

(A)

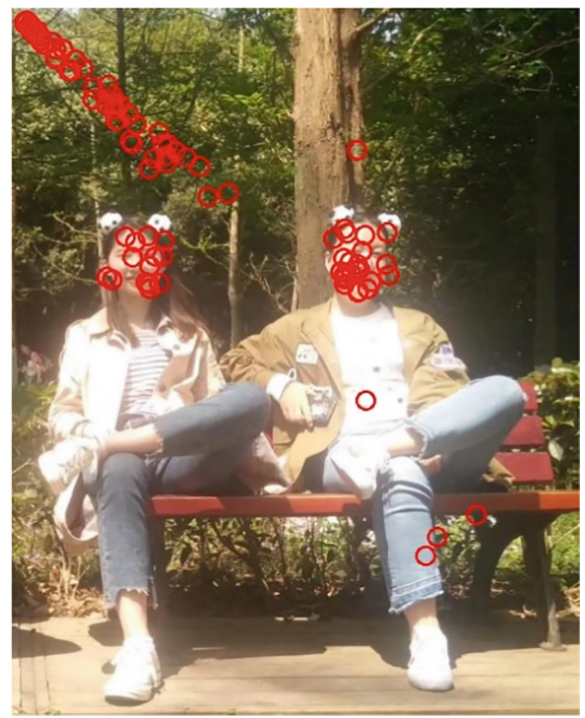

(B)

Fig. 8. The attention distribution generated by Attention Strategy Controller.

\section{Conclusion}

In this paper, we have proposed a bionic cascaded framework for face detection in service robots. Experimental results demonstrate that our methods are effective. The proposed algorithm is easy to run on an embedded system adopting the design of cascaded detector. The system can achieve real time face detection, and simulate the attention mechanism of human beings, which can improve the performance of interaction pattern. Combining the characteristic of service robot systems, the proposed design balance the power consumption, real-time performance, user experience etc. In the future, we will exploit more eye tracking data to train the system, to further improve the performance.

Acknowledgement. This research is supported by the Doctoral Scientific Research Fund of Hubei University of Technology (No. BSQD2017071).

\section{References}

1. Viola, P., Jones, M.: Robust real-time face detection. Int. J. Comput. Vis. 57(2), 137-154 (2004)

2. Yang, B., et al.: Aggregate channel features for multi-view face detection, pp. 1-8 (2014)

3. Pham, M.T., et al.: Fast polygonal integration and its application in extending haar-like features to improve object detection. In: The Twenty-Third IEEE Conference on Computer Vision and Pattern Recognition, June 2010, CVPR 2010, pp. 13-18. IEEE, San Francisco (2010) 
4. Zhu, Q., et al.: Fast human detection using a cascade of histograms of oriented gradients. In: 2006 IEEE Computer Society Conference on Computer Vision and Pattern Recognition. IEEE (2006)

5. Mathias, M., Benenson, R., Pedersoli, M., Van Gool, L.: Face detection without bells and whistles. In: Fleet, D., Pajdla, T., Schiele, B., Tuytelaars, T. (eds.) ECCV 2014. LNCS, vol. 8692, pp. 720-735. Springer, Cham (2014). https://doi.org/10.1007/978-3-319-10593-2_47

6. Yan, J., et al.: The fastest deformable part model for object detection. In: 2014 IEEE Conference on Computer Vision and Pattern Recognition (CVPR). IEEE Computer Society (2014)

7. Zhu, X., Ramanan, D.: Face detection, pose estimation, and landmark localization in the wild. In: 2012 IEEE Conference on Computer Vision and Pattern Recognition (CVPR). IEEE (2012)

8. Krizhevsky, A., Sutskever, I., Hinton, G.: ImageNet classification with deep convolutional neural networks. In: Advances in neural information processing systems, vol. 25, no. $2(2012)$

9. Sun, Y., Wang, X., Tang, X.: Deep learning face representation by joint identificationverification (2014)

10. Yang, S., et al.: From facial parts responses to face detection: a deep learning approach (2015)

11. Li, H., et al.: A convolutional neural network cascade for face detection. In: 2015 IEEE Conference on Computer Vision and Pattern Recognition (CVPR). IEEE Computer Society (2015)

12. Zhang, K., et al.: Joint face detection and alignment using multitask cascaded convolutional networks. IEEE Signal Process. Lett. 23(10), 1499-1503 (2016)

13. Yonghao, H., Dezhong, X., Lifang, W., Meng, J., Shiming, X., Chunhong, P.: LFFD: a light and fast face detector for edge devices. https://arxiv.org/abs/1904.10633. Accessed 26 Apr 2019

14. Freeman, W.T., Adelson, E.H.: The design and use of steerable filters. IEEE Trans. Pattern Anal. Mach. Intell. 13(9), 891-906 (2002)

15. Shiwei, C., Lingyun, S.: A survey on visualization for eye tracking data. J. Comput.-Aided Design Comput. Graphics 26(5), 698-707 (2014). (In Chinese) 